trout farms use about 30,000 gallons of high quality fresh water for each pound of fish produced and the availability of sites for expansion is very limited. Two methods for overcoming this constraint are the use of recycling systems and the culture of trout in sea water. The former can be regarded as a technological development based on present practices, whereas sea-water culture is a more radical change with many facets requiring investigation. Although no major research work on salmonid farming has been undertaken by MAFF or the Department of Agriculture and Fisheries for Scotland some facilities are available and plans for additional facilities are in hand: in these, special attention will be directed towards nutritional studies, cage systems in the sea, recirculation systems for fresh and salt water, and genetic studies aimed at producing improved strains. Disease research and monitoring is already covered both in England and Wales and in Scotland.

Flatfish farming research has not yet established a sufficiently reliable technology for full commercial development. The first priority for further work lies with turbot and especially with the need to overcome the problems of hatchery production of juveniles.

One major area of work of fundamental importance to any form of fish farming is the development of a genetic approach to produce improved strains, to avoid inbreeding depression and to conserve genetic uniformity and hence performance in domesticated lines of fish. A further useful field with genetic implications is the development of allyear-round spawning stocks and the control of sex itself. Genetic manipulation at the hatchery level is at present studied within the MAFF programme, but no facilities exist for extending this to the growing-on phase or for holding stocks of domesticated fish. For marine flatfish, the creation of domesticated lines has not yet begun. Similarly, Atlantic salmon stocks are basically of wild origin but rainbow trout exist in a wide variety of selected lines, particularly in North America. Although a proper evaluation of their merits, and the possibility of heterosis in F1 hybrids, seems not to have been attempted, this is an area of study which could have an immediate beneficial effect on farming practices in the UK.

In conclusion, there is an established and expanding industry for salmonid farming, principally in fresh water, and a growing interest on the part of large companies in marine flatfish culture. In view of the likely diminution of supplies of prime quality fish like sole and turbot from the sea it is desirable to encourage the continued expansion and development of this area of food production, on a broad basis and at all levels of commercial involvement, though this should not be construed as implying any substantial contribution to offset a short-fall in basic supplies. To this end the programmes of research and development in the fields of hatchery production, environmental control, nutritional innovation, genetic manipulation and disease identification and control within the species at present exploited or showing potential for exploitation require careful integration. The list of species at present includes rainbow trout, salmon and turbot with secondary consideration for brown trout, sea trout and sole.
THE term "vitamin(e)" was coined by Casimir Funk in 1912. He was good at promoting its use, but he could never have foreseen the mind-expanding effect of this new word in the 1970s. Vitamins are coenzymes and other metabolic catalysts whose pathways of biochemical synthesis have been lost as a result of mutations during the evolution of animals. Identification and synthesis of vitamins led to spectacular successes in preventive medicine; these became well known, and, as one result, popping vitamin pills is part of the American way of life, just like colour television in the family room. The idea is not so much that diets are deficient, but why not take vitamins "just to be safe"? Indeed, except for a half-hearted attempt some years ago by the US Food and Drug Administration (FDA) to throw cold water on it, the habit had few opponents and it was certainly better than smoking cigarettes.

There is, however, a basic pharmacological delusion of the layman that if a small dose of a medicine is good, a large dose must be even better, especially if it is not poisonous. Many physicians have treated patients whose symptoms exceeded in severity their physical diagnosis, by prescribing "a good rest with lots of vitamins". This procedure is known as "placebo therapy". the administration of a is that such dosage will produce superharmless nostrum for soothing the health, an effect not measurable in nerves of the apprehensive, and for human beings or laboratory animals.
convincing those who are open to Vitamins go through periods of convincing those who are open to

\section{Funk therapy}

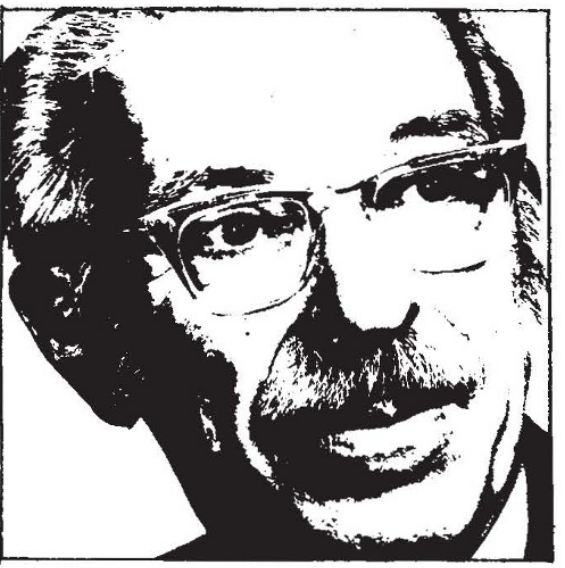

THOMAS H. JUKES

suggestion that they are receiving "medical treatment". Vitamins can be sold without prescription, and the medical profession, by fostering the use of vitamin pills, made an opening for nonphysicians to practice medicine without a licence. The "health-food" industry responded, and its sales outlets stock pills containing vitamins and alleged vitamins. If health food is so "nutritious", why does it need supplementation with large doses of vitamins and minerals? The answer given in the health food literature fashion. Thiamine was in the spotlight at one time and, more recently, ascorbic acid became the darling of the headlines. Vitamin $\mathbf{B}_{12}$ is popular, probably because it is often injected and is red. Vitamin $\mathbf{B}_{6}$ is all the rage now, and vitamin $\mathrm{E}$ is a hardy perennial because it was lucky enough to be discovered by the fact that its deficiency causes infertility in rats. The same is true of vitamin A, of course, but $\mathrm{E}$ has ever since worn the halo of Aphrodite, and has collected many unearned credits.

"Megavitamin therapy" has its devotees, and publicity is currently given to "orthomolecular psychiatry", defined as the "achievement and preservation of good mental health by the provision of the optimum molecular environment for the mind, especially the optimum concentrations of substances normally present in the human body, such as the vitamins". Whether or not this actually works we shall probably never know, because the author (Linus Pauling) also says "the principles of medical ethics prevent orthomolecular psychiatrists from withholding from half of their patients a treatment that they consider to be valuable. Controlled tests can be carried out only by skeptics".

Efforts of the FDA to regulate megavitamin promotion, however, were set back by a court decision and by the passage last year of a bill in the US Senate that specifically prevents the FDA from classifying highpotency vitamin preparations as drugs. 